\title{
The 45th Annual Meeting of the European Society for Blood and Marrow Transplantation: Nurses Group - Oral Session
}

C Springer Nature Limited 2019

24-27 March 2019 Frankfurt, Germany

Modified and published with permission from https://www.ebmt.org/annual-meeting

Sponsorship Statement: Publication of this supplement is sponsored by the European Society for Blood and Marrow Transplantation. All content was reviewed and approved by the EBMT Committee, which held full responsibility for the abstract selections.

Presenting author names are bold type in the contributor lists.

\section{NO001}

Re-engineering follow-up care after allogeneic stem cell transplantation: Patients' and clinicians` perspectives of ehealth enhanced support care - The smile study

Sabine Valenta ${ }^{1,2}$, Lynn Leppla ${ }^{1,3}$, Alexandra Teynor, Anja Schmid ${ }^{3}$, Robert Zeiser ${ }^{3}$, Monika Engelhardt ${ }^{3}$, Sabina De Geest ${ }^{1,5}$

${ }^{1}$ University Basel, Institute of Nursing Science, Department
Public Health, Basel, Switzerland, ${ }^{2}$ University Hospital
Basel, Hematology, Basel, Switzerland, ${ }^{3}$ Freiburg
University Medical Center, Departments of Hematology
and Oncology, Freiburg im Breisgau, Germany, ${ }^{4}$ University
of Applied Sciences Augsburg, Faculty of Computer
Science, Augsburg, Germany, ${ }^{5}$ KU Leuven, Academic
Centre for Nursing and Midwifery, Department of Primary
Care and Public Health, Leuven, Belgium

Background: Re-engineering follow-up care after Hematopoietic Stem Cell Transplantation (HSCT) is crucial due to an increasing number of long-term survivors in need of comprehensive care. eHealth solutions show promise in improving clinical outcomes in cancer patients. Yet successful implementation and sustainability of innovative eHealth supported care models requires application of implementation science methods including contextual analysis. We report the contextual analysis as part of the development, implementation and testing of the effectiveness of an Integrated model of care in allogeneic hematopoietic $\underline{S}$ teM cell transplantatIon faciLitated by eHealth (SMILe) in view of

(1) HSTC patients' and clinicians`current experiences of follow-up care,

(2) their perception of eHealth-enhanced follow-up care and (3) patients' technology openness.

Methods: We applied a single-centre explanatory sequential mixed methods design (QUAN: questionnaires; QUAL: interviews). Patients were recruited from outpatient clinic of University Medical Centre Freiburg, Germany, who were six weeks to three years after allogeneic HSCT. Data were collected from 06/2017-01/2018. QUAN: We surveyed a convenience sample of 60 adult HSCT patients. Previously developed methodology was applied to assess patients' and clinicians ' current experiences of follow-up care (BRIGHT-study) and technology openness (PICASSOTX study). Descriptive statistics were used to explore means, medians and distribution of the data. QUAL: The quantitative results informed the sampling procedure of semi-structured interviews with 10 patients as well as three focus groups with clinicians. The interview guide was developed by using dimensions of the eHealth-enhanced Chronic Care Model. Qualitative data were analysed by thematic analysis.

Results: (1) Current clinical practice is mostly acute care driven with less focus at self-management support, since self-management support was rarely provided (10-15\%). The hospital-home transition was characterized by 
insecurity, with a perceived need for medical monitoring and decision support to control fear for relapse, infections or new symptoms. Clinicians observed problems in patients' decision making and adherence to recommended actions.

(2) eHealth was seen as important source of support, but should not replace human contacts. A permanent connection with the transplant center via eHealth was perceived as valuable to reduce insecurity, to support recognizing, judging and acting upon new symptoms, and to provide behavioral support (e.g. medication adherence or infection prevention). Clinicians also observed patients' problems in recognizing and judgement of symptoms. They highlighted the need for nurse counselling and regular eHealth supported follow-up care.

(3) Most patients $(70 \%)$ were open towards new technologies and all of them (100\%) already used technologies (e.g. computers, smartphones) to get information about health problems. Patients rated the importance of developing new technologies to allow clinicians to monitor their health behaviors, symptoms and other medical parameters with a median of 8 (scale $0-10$ ). Knowing that a clinician is overlooking their data would increase feelings of security (63\%).

Conclusions: Our findings highlight the need of reengineering follow-up care after HSCT, since current clinical practice is mostly acute care driven and both, clinicians and patients, expressed the need for an eHealth enhanced self-management support after HSCT. Our findings set the stage for the development and implementation of an eHealth-powered integrated model of care in HSCT.

Disclosure: Nothing to declare.

\section{NO002}

\section{Training and education on infection prevention and} control in nursing practice in low and middle income countries - what is needed?

\section{Aleksandra Babic ${ }^{1}$, Jacqui Stringer ${ }^{2}$, Sarah Liptrott ${ }^{3}$, Sulman Siddique ${ }^{4}$, Steffie van de Werf ${ }^{5}$, Christophe Peczynski $^{6}$, Iris Agreiter ${ }^{7}$, John Murray ${ }^{8}$}

${ }^{1}$ Oncology Institute of Southern Switzerland, Transplant Unit, Bellinzona, Switzerland, ${ }^{2}$ Christie Hospital NHS Foundation Trust, Manchester, United Kingdom, ${ }^{3}$ IEO, European Institute of Oncology IRCCS, Milan, Italy, ${ }^{4}$ Centre for Innovative Research Across the Life Course (CIRAL), London, United Kingdom, ${ }^{5}$ Leiden University Medical Center, Leiden, Netherlands, ${ }^{6}$ Hôpital Saint Antoine, Paris, France, ${ }^{7}$ HODC St. James's Hospital, Dublin, Ireland, ${ }^{8}$ Christie Hospital NHS Foundation Trust, Haematology and Transplant Unit, Manchester, United Kingdom
Background: Infection is a significant cause of mortality in post haematopoietic stem cell transplantation (HSCT) globally. Low and middle income countries (LMICs) are particularly at risk because of a documented lack of adequately qualified infection prevention and control (IP\&C) professionals and appropriate guidelines.

Methods: An online questionnaire was developed using JACIE's minimum standards for IP\&C in BMT centres. The anonymized survey has been developed by the EBMT global educational committee in collaboration with the EBMT research committee and infectious diseases working party, and was distributed at a single time point. Email addresses were kindly provided by Nurses No Frontiers Association, Childhood Cancer International and WBMT. Forty questions addressed the following domains: nursing education and training, hand hygiene, personal protective equipment (PPE), central line associated infection, general hygiene and antibiotic stewardship.

Results: Data from 19 countries was obtained, a total of 72 BMT centres answered, 63 replies were valid for statistical analysis. Most of the questionnaires were completed by nurses $(59,7 \%)$, followed by doctors $(35,5 \%)$ and other personnel in $4,8 \%$ of centres. $89 \%$ of centres reported having an infection control (IC) nurse in the unit, who in $47,5 \%$ of cases received certified IC training. Written IC policies and procedures are available in $91,5 \%$ of facilities, $83,3 \%$ of them are evidence based and $78,3 \%$ are updated and reviewed annually. Education on IP\&C for patients and family members is carried out mainly by nurses $(90,5 \%)$, with doctors delivering the education in $66,7 \%$ of cases. Education is primarily delivered during face to face sessions (95,2\%). $22 \%$ of centres supplement this with web based videos, $73 \%$ of centres use written material. Training on central venous catheters is competency-based in $91,5 \%$ of centres and $84,5 \%$ of personnel can demonstrate competency with maintenance. Standard precautions and PPE have specific policies in $93,2 \%$ of centres, get job specific training at time of employment in $93,2 \%$ of hospitals, are audited in $86,2 \%$ of BMT units and validated every 12 months in $74,1 \%$ of cases Antimicrobial stewardship training to nurses is given by $47,5 \%$ centres, $85 \%$ of the BMT units have written policies on antibiotics and $89,8 \%$ of facilities declare to have an active collaboration with the microbiology laboratory. Training in IC for nurses has been supported in $81 \%$ of BMT units and $62,5 \%$ of nurses are aware of its importance. 56,1\% of nurses got IC training during the last 6 months, $12,3 \%$ during the last year, 22,8\% cannot remember and $8,8 \%$ has never had IC training. The most preferred method on IC training is on the job $41,4 \%$, $27,6 \%$ of nurses like web based training, $15,5 \%$ in classroom, Off site workshops are preferred by $12,1 \%$ and $1,7 \%$ are happy with distance learning. 
Conclusions: Education and training are the core items of the survey. However, it appears there are policies and training in place in most centres. Most important learning methods are face to face education and training on the job. Further investigation is needed to explore if nurses in centres without standardized training might benefit form certified IC training based on JACIE standards indication.

Disclosure: None

\section{NO003}

CAR-T cell therapy in an ambulatory care (AC) UNIT: The impact on nursing practice

\section{April Nesbitt $^{1}$, Diana Comerford ${ }^{1}$, Leigh Wood $^{2}$, Claire Roddie $^{3}$, Karl Peggs ${ }^{3}$}

${ }^{1}$ University College London Hospital, Ambulatory Care, London, United Kingdom, ${ }^{2}$ University College London Hospital, Haematology Trials, London, United Kingdom, ${ }^{3}$ University College London Hospital, Haematology, London, United Kingdom

Background: AC delivers inpatient chemotherapy and supportive care treatments to patients fulfilling clinical criteria. Patients stay at home or in a hotel and attend AC daily for assessment and treatment. Unwell patients are admitted to an inpatient haematology ward. AC has been delivering haematopoietic stem cell transplants (HSCT), autologous and allogeneic, since 2005. When CAR-T cell therapy trials commenced in 2016, suitable patients were treated in AC because this setting offers clinically stable patients a degree of independence during their admission. A strict minimum length of stay is mandated per trial protocol for safety monitoring. AC provides specialist CAR-T knowledge with regard to infusions and supportive care since the unit treats a large number of HSCTs. A retrospective audit was conducted to evaluate how CAR-T cell therapy has affected nursing practice within $\mathrm{AC}$, and why patients were admitted from AC to hospital.

Methods: Patients that had CAR-T therapy in AC at UCLH between September 2016 and August 2018 were identified and causes of admission to inpatient were examined using Filemaker. Chemocare was used to review chemotherapy protocols for each trial.

Results: Trial treatments were given to treat relapsed and/or refractory: lymphoma (COBALT, ALEXANDER AUTO 3); leukaemia (ALLCAR19, CARD); and myeloma (APRIL AUTO 2). Twenty-one patients commenced treatment in AC. Two patients were admitted as part of trial protocol, and 12 unplanned admissions: nausea/ vomiting (3), pyrexia (7), monitoring (1) and moisture lesion (1). The remainder were discharged home direct from AC. All trial conditioning regimes comprised of fludarabine and cyclophosphamide, ALLCAR19 also required pre and post hydration.

Conclusions: Delivering CAR-T cell therapy has minimally impacted nursing practice in AC. Twenty-one patients over a 23 month period is not a large number to absorb into a service. Challenges have included increased administrative workload to arrange new trial blood protocols, learning about CAR-T cells and associated toxicities, and working with a newly established trials team to understand each other's way of working to coordinate care, including times for cell return as per trial protocol. The number of cell returns per day is limited to balance workload and takes into account other HSCT patient groups.

Fludarabine and Cyclophosphamide are given frequently in $\mathrm{AC}$, and 24-hour infusions are delivered through a portable infusion pump via central venous access where needed. During CAR-T cell infusions, a physician and research practitioner from the trials team were present to record necessary data, allowing AC nurses to focus on cell return.

No unexpected adverse events happened to these patients during their stay in AC, and reasons for unplanned admissions were anticipated due to them having had chemotherapy. However, these reasons could also be signs of CAR-T specific toxicities. Daily CAR-T specific assessments (to monitor for neurotoxicity and cytokine release syndrome) were completed by the trials team. AC nurses have since been trained to undertake this assessment twice daily. This allowed the AC team to be more involved in caring for patients undergoing CAR-T therapy improving continuity of care providing greater autonomy within a nurse-led service.

Disclosure: Nothing to declare

\section{NO004}

'GITMO 1000 miles': A national nurses group initiative taking education to nurses

Laura Orlando $^{1,2}$, Iris Agreiter ${ }^{2,3}$, Stefano Botti ${ }^{2,4}$, Marta Canesi $^{2,5}$, Diana Cerne ${ }^{2,6}$, Marco Cioce ${ }^{2,7}$, Valentina De Cecco $^{2,8}$, Letizia Galgano ${ }^{2,9}$, Sarah Jayne Liptrott ${ }^{10}$, Antonio Magarò $^{1,2}$, Cristina Mentone ${ }^{2,11}$, Anna Rita Pesce $^{2,12}$, Emanuela Samarani ${ }^{2,13}$, Sonia Soave ${ }^{2,14}$, Angela Lina Trunfio ${ }^{2,15}$, Francesca Bonifazi ${ }^{2,16}$, Gianpaolo Gargiulo ${ }^{2,17}$

${ }^{1}$ IEO, European Institute of Oncology IRCCS, Oncohematology Department, Milano, Italy, ${ }^{2}$ on behalf GITMO, Genova, Italy, ${ }^{3}$ St. James's Hospital, HODC, Dublin, Ireland, ${ }^{4}$ Arcispedale Santa Maria Nuova-IRCCS, Haematology Unit, Reggio Emilia, Italy, ${ }^{5}$ Fondazione MBBM ASST, Department of Pediatric Haematology and 
Transplant Unit, Monza, Italy, ${ }^{6}$ Azienda Sanitaria Universitaria Integrata, Haematology and BMT Unit, Udine, Italy, ${ }^{7}$ Founadtion Policlinico Universitario A. Gemelli IRCCS, Rome, Italy, ${ }^{8}$ Policlinico S. Matteo Pavia, Paediatric Haemato-Oncology Unit, Pavia, Italy, ${ }^{9}$ Azienda Ospedaliera Universitaria Careggi, BMT Unit Department of Haematology, Florence, Italy, ${ }^{10}$ IEO, European Institute of Oncology IRCCS, Oncohematology Department, Milan, Italy, ${ }^{11}$ Mauriziano Umberto I Hospital, University Division of Haematology, Turin, Italy, ${ }^{12}$ 'SS. Antonio e Biagio e Cesare Arrigo' Hospital, Haematology and BMT Unit, Alessandria, Italy, ${ }^{13}$ Spedali Civili Brescia, BMT Unit, Brescia, Italy, ${ }^{14}$ 'Tor Vergata' University, Division of Haematology, Rome, Italy, ${ }^{15}$ ASST Papa Giovanni XXIII, Haematology Unit, Bergamo, Italy, ${ }^{16} \mathrm{~S}$. Orsola-Malpighi University Hospital, University of Bologna, Bologna, Italy, ${ }^{17}$ AOU Federico II Napoli, Haematology and BMT Unit, Naples, Italy

Background: The JACIE standards (version 7) point B3.7 require that the Clinical Program shall have nurses formally trained and experienced in the management of patients receiving cellular therapy. It also requires that nurses shall have received specific training and maintain competence in transplant-related skills that they routinely practice (B.3.7.3). One objective of the GITMO Nurses Group (Italian Group of Bone Marrow Transplant), is training and dissemination of evidence based knowledge to nurses working in the transplant setting. Unfortunately, the number of national training events cannot completely cover the training needs of the 100 transplant centers across Italy. In order to promote a more capillary approach the GITMO Nursing Group devised a project allowing training to be brought to Italian hospitals: door-to-door by expert peers.

Objectives: To promote the acquisition and maintenance of JACIE oriented skills; To reach the greatest number of nurses operating in the field of transplantation at a national level; and Shorten distances and allow professional training and updating based on best practices.

Methods: The GITMO Nurses Group shared and approved the "GITMO 1.000 miles" training program defining eight events per year. The topics covered were based on the skills described in paragraph B3.7 of JACIE standards for nurses. Each course last approximately four hours with the possibility to extend to eight hours. The host centre collaborates closely with the GITMO Nurses Group on the program draft, selecting topics according to local needs. All courses are CME accredited through a single national provider. A CME questionnaire, based on national standards, makes it possible to evaluate participants learning, whilst a satisfaction questionnaire is given to participants to evaluate the relevance, quality and usefulness of the event, using a 5 item Likert scale.
Results: From December 2016 to September 2018, fifteen centres have been involved: six in the north of Italy, three in the centre and six in the south and islands. 1283 nurses participated, 432 in the north, 321 in central and 530 in southern Italy (including islands). $89 \%$ of participants passed the learning tests. In terms of satisfaction, results achieved were medium to high. The course was considered relevant in $92 \%$ of cases (1180 learners), of good quality in $93 \%$ of cases (1193 learners) and really useful in $92 \%$ of cases (1180 learners) (Figure 1).

Conclusions: Guaranteeing and promoting educational initiatives is one of the principal objectives of scientific societies, however, in terms of resources and organization it is not always possible to produce a tailored format suitable for all. The diversity of participants needs to be considered in terms of existent competencies and in terms of professional development as well as the possibility for ad hoc course development. Interpreting education needs and taking education 'home' (as in the homing phenomenon of stem cells) is not easy, but this project has been able to combine many factors. 1283 professionals were involved with a high score (89\%) that has allowed the realisation of teh JACIE standard JACIE in terms of acquisition and maintenance of competencies.

Disclosure: Nothing of declare

\section{NO005}

Implementation of an adult nurse-led stem cell donor search and long term follow-up process Ghent university hospital

\section{Johan De Munter ${ }^{1}$, Marijke Quaghebeur ${ }^{2}$, Sofie De Wilde $^{1}$, Evi Sprangers ${ }^{2}$, Amy Senave ${ }^{2}$, Anna Vantilborgh ${ }^{2}$, Philip Vlummens $^{2}$, Tessa Kerre ${ }^{2}$}

${ }^{1}$ University Hospital Ghent, Cancer Center, Ghent, Belgium, ${ }^{2}$ University Hospital Ghent, Hematology Department, Ghent, Belgium

Background: Published reports on the outcome after allogeneic hematopoietic stem cell transplantation (HSCT) have only indirectly mentioned the consequences of donation for the matched related donor (MRD) or matched unrelated donor (MUD). The importance of donor care management before and during the donation process, and long term follow-up of stem cell donors has become increasingly evident. It's obvious that the donor receives clear and correct information, undergo correct screening procedures and data collection is done correctly. The psychosocial impact on the potential stem cell donor should not be underestimated. These donor needs stressed the importance to have a standardized nurse-led counselling, starting from the 
donor search, during the donation process and through donor follow-up.

Methods: An inter-professional HSCT working group was assembled for developing an evidence based care standard operating procedure (SOP) for donor care management, compatible with the current JACIE standards. In this process, different types of staff shared evidence-based knowledge, expertise and skills to positively impact the donor care management outcomes.

Results: The implementation of the SOP increased the structure in the donor care management for MRD and MUD donors and pointed out the responsibilities to all healthcare providers within the hematology department. The new way of working improved the collaboration between the interprofessional stem cell transplantation team, physicians in training and the specialized nurses, which is expected to improve donor care outcomes. The nurse-led donor clinic also improves accessibility and screening of MRD donors via daily consultation times. This ensures that family donors can sign up quickly, discuss their concerns immediately and will receive systematic feedback about the results of the screening procedure. The nurse-led stem cell donor clinic guarantees complete confidentiality to all donors, as prescribed in the applicable JACIE standards.

The introduction of the nurse-led donor clinic resulted in the creation of structured MRD/MUD database, currently functioning as the basis of the long term donor follow-up process.

Accurate data collection of long term follow-up in MRD and MUD donors is time-consuming. Using a telephone/ mail-based long term follow-up often leads to out of date data or donors becoming lost to follow-up. To optimize the donor screening and long term data collection, a digital stem cell donor care pathway was recently introduced. This digital pathway runs through a secure patient website and is connected with the hospital electronic patient record. It offers access to a variety of functions including secure messaging, protected donor information, appointment scheduling and/or donor questionnaires.

Conclusions: Inter-professional pathways are needed to establish systems to adequately address donor care and facilitating comprehensive donor counselling to all stem cell donors. The nurse-led donor clinic is effective in providing donor-tailored information, assures all necessary screening tests are performed as described in the SOP, and long term follow-up is done according to JACIE standards and international guidelines.

Adding a digital donor care portal to the already existing follow-up systems can further optimize the donor care management. Future evaluation on the donor satisfaction will be needed to evaluate the effects of this new donor care pathway.

Disclosure: Nothing to declare

\section{NO006}

Feasibility of apheresis procedure for children $\&$ adolescence in preparation for CAR T-cell treatment

\section{Daphna Hutt ${ }^{I}$, Bella Bielorai ${ }^{1,2}$, Bella Baturov ${ }^{I}$, Ina $Z^{\prime}$ 'orbinski ${ }^{1}$, Natalia Ilin ${ }^{1}$, Michal J. Besser ${ }^{2,3}$, Amos Toren $^{1,2}$, Elad Jacoby ${ }^{1,2}$}

${ }^{1}$ Sheba Medical Center, Pediatric Hem-Onc \& BMT, Ramat-Gan, Israel, ${ }^{2}$ Sackler Faculty of Medicine, Tel Aviv University, Tel Aviv, Israel, ${ }^{3}$ Sheba Medical Center, Ella Lemelbaum Institute of Immuno- Oncology, Ramat-Gan, Israel

Background: Autologous CD19 CAR-T cells demonstrate remarkable remission rates in relapsed and refractory acute lymphoblastic leukemia (R/R-ALL) and B-cell lymphoma, with best results achieved in pediatric patients. CAR-T cells are manufactured from a leukapheresis product, of which $\mathrm{T}$-cells are activated, genetically modified and expanded. Pediatric apheresis procedures are considered safe but challenging given the small blood volume of children, insufficient cell numbers in heavily pretreated patients, and need of adequate venous access.

Methods: Between 5/2016 and 10/2018, 27 pediatric patients with R/R-ALL $(n=23)$ and B-cell lymphoma $(n=$ 4) were leukapheresed for CAR-T cell manufacturing in a locally production-based clinical trial (NCT\#02772198). Leukapheresis was performed at the pediatric outpatient clinic in a familiar atmosphere and coordinated and led by specialized nurses. Age-appropriate preparation was provided to patients and families prior to procedure in order to enhance cooperation.

Lymphocyte collection efficiency (LCE) was calculated using T-cell count in the blood prior to apheresis and T-cell count in the apheresis product. Initial production was planned for $4 \times 10^{8}$ cells per patient. The target dose for administration was $1 \times 10^{6} \mathrm{CAR}-\mathrm{T}$ cells $/ \mathrm{kg}$ recipient. Data regarding apheresis, cellular production and outcomes were analyzed.

Results: In 27 enrolled patients, the median age was 9 years (range 3-22) and median weight 34kg (range 15-69). A single leukapheresis procedure was performed in all patients. Priming with blood was needed only for one patient, weighing $15 \mathrm{~kg}$. Leukapheresis was preformed using peripheral vein access in 23 of the 27 patients. The remainders had access via arterial lines $(\mathrm{n}=3)$ and central venous catheter $(\mathrm{n}=1)$. The average procedure length was 192 minutes (range 114-295). No severe adverse events were recorded. LCE was variable (ranged 2-60\%), and was below $10 \%$ in 5 patients. In 3 patients, the apheresis did not achieve initial cell requirement, and they had an LCE of $17 \%, 22 \%$ and $2.5 \%$. Despite this, target dose for 
administration was achieved in all 3 patients and they had a clinical response to the CAR T-cell treatment. Overall, 24 patients received CAR-T cell product: 23 at the desired dose and 1 in a lower dose $\left(0.8 \times 10^{6} / \mathrm{kg}\right)$. Two patients died prior to lymphodepletion from disease progression and one had production failure. Of the 24 patients treated, 21 responded to CAR-T cells, including the one patient who did not achieve the target cell dose.

Conclusions: Pediatric leukapheresis for CAR-T cells production was well tolerated and safe. Collection of sufficient amount of T-cells in heavily pretreated pediatric patients is feasible even with relatively low LCE, with no side effects. Nurses play a major role in the preparation and management of the procedure.

Clinical Trial Registry: Clinicaltrials.gov

NCT\#02772198

Disclosure: Nothing to declare

\section{NO007}

The value of rehabilitation interventions to address the needs of teenage and young adult oncology patients post haematology transplant

\section{Anna Mackland ${ }^{1}$, Nicola Chesman ${ }^{2}$}

${ }^{1}$ The Christie Hospital NHS Foundation Trust, Manchester, United Kingdom, ${ }^{2}$ The Christie NHS Foundation Trust, Manchester, United Kingdom

Background: On average 18 Teenage and Young Adult (TYA) patients (16-24) are admitted to The Christie for stem cell transplants a year. Research highlights that physical and psychological issues can be common and prolonging for a young person during and following a transplant (Cooke et al, 2011). The Christie has a dedicated TYA Occupational Therapist and Physiotherapist who provide rehabilitation interventions to TYA patients during their cancer treatment and beyond. There is limited research available specifically focused on TYA transplant needs and the value of rehabilitation interventions for this age group. The Physiotherapist and Occupational Therapist identified that there were needs of the TYA patients that they could help to address.

Methods: The Occupational Therapist and Physiotherapist completed an 8 week pilot to attend the weekly adult post-transplant clinic. TYA's were approached individually to explain the rehabilitation service and if they consented their needs were discussed via a Holistic Needs Assessment (HNA). Rehabilitation interventions were provided at the time or an additional appointment was made. Data was recorded in regards to numbers of patients seen, time spent with patients and interventions provided.
Results: 15 TYA patients attended the clinic during the 8 week pilot. 12 patients were approached (1 patient not appropriate, 2 patients missed due to early appointments). Only 1 patient declined rehabilitation interventions. $67 \%$ of TYA patients engaged with rehabilitation interventions.

The HNA's completed highlighted specific difficulties with memory/concentration difficulties, fatigue, eating/ appetite changes, sleep problems or nightmares and difficulty with getting around/ walking. Emotional concerns were also highlighted as a difficulty with $50 \%$ of TYA's reporting feelings of worry, fear or anxiety, and also reporting feelings of anger/frustration, loss of interests in activities, feelings of sadness/depression and difficulty making plans. Alarmingly $50 \%$ of patients scored over 5 on the concerns scale with 1 patient scoring 9/10 and had not discussed this in the clinic appointment.

During the pilot the Physiotherapist provided a range of interventions including exercise advice, exercise bike referral, oedema advice, breathlessness management, muscular skeletal assessment and exercises. The Occupational Therapist provided advice on sleep management, anxiety management, return to work/education signposting, and interventions to support memory/concentration. Both therapists provided activity advice, fatigue management, referrals to other services and engaged in supportive conversations to improve quality of life.

There were barriers identified to the pilot including limited space in the busy clinic for private conversations, and the length of the pilot also did not give sufficient time to re- evaluate HNA results but patients self-reported that they had found the interventions helpful and would engage with services in the future.

Conclusions: The pilot has highlighted a need for a specific TYA transplant clinic to address the significant physical and psychological needs of this age group and the value of rehabilitation interventions to address these needs. There is also an identified need for a dedicated TYA transplant support group. A larger scale pilot (6 months) will commence in November 2018 in order to evaluate the effectiveness and impact of these interventions with a larger cohort.

Disclosure: Nothing to declare

\section{NO008}

The impact of bone marrow transplant process for a family with five siblings, four requiring a BMT and one HLA matched and donor for all

\section{Joanne Ellis ${ }^{1}$, Robert Francis Wynn ${ }^{2}$}

${ }^{1} \mathrm{RMCH}, \mathrm{BMTU}$, Manchester, United Kingdom, ${ }^{2} \mathrm{RMCH}$, Manchester, United Kingdom 
Background: Often in bone marrow transplant (BMT) in genetic disease, there is a requirement to transplant more than one family member. This impacts significantly on the BMT experience for families, and health care professionals.

Manchester is a BMT center for metabolic diseases and in 2016 were referred 4 siblings with Mitochondrial NeuroGastro-Intestinal Encephalopathy (MNGIE). MNGIE is an autosomal recessive disorder in which thymidine pohphoylase mutation leads to progressive neuropathy, particularly of the gut, and eventual intestinal failure and death. BMT works by providing the deficient enzyme and halting disease progression.

A single, unaffected sibling was HLA matched with all 4 of the affected siblings and a decision was made for her to donate. All 4 patients were transplanted between 2016 and 2018 with the sibling donor and this paper looks at the process and co-ordination of transplanting siblings and the effects of BMT on the family.

Methods: The process for the sibling donor was commenced and referral for HTA assessment and independent medical assessment was made. Several Pre-BMT discussions took place via an interpreter due to the parents being non English speaking. Support and advice were given to family after MDT and planning meeting as to the order of the siblings to be transplanted and the process for the donor. Once cleared by HTA and IM the donor was referred for PBSC collection, vascath insertion. PBSC were collected and split into 4 doses for each sibling then frozen. The prioritisation of the siblings, the use of the single healthy sibling and the hospitiasations of the affected children all impacted on family life. Transplant process occurred with the eldest first, once discharged the next sibling in age order with the last 2 children being admitted at the same time and transplanted within a week of each other, was due to family needs and requirements.

Now all 4 children are well and at home, we review the impact of the transplant process on the physical and psychological health of the donor and recipients, and the wider family. We review the literature of multiple transplants within the same family.

Results: The transplants have been a success and all 4 children discharged home, although the elder 2 siblings remain on PN. The younger 2 have continued enteral and oral feeding, as the disease was less advanced. There has been disruption to normal family life, including to the life of the healthy donor. We review the physical, psychological and social functioning of parents and their 5 children.

Conclusions: The co-ordination and support required for such a complex family requires lots of time, planning and meeting with the family, listening and advocating their needs. The whole MDT team are heavily involved but a central person is required to liaise with the family, this being the nurse specialist. Both positive and negative comments have been given during an interview with the family with the intention to improve the service should this happen in the future.

Disclosure: Nothing to declare

\section{NO009}

A prospective approach of migrants and ethnic minorities in order to overcome poor adherence and to guarantee an equal access to innovative transplant procedures

Sandra Michiels ${ }^{1,2}$, Dominique Bron ${ }^{3}$, Patrick Crombez ${ }^{3}$, Nathalie Meuleman ${ }^{3}$, Fati Kirakoya ${ }^{2}$, Sandra TricasSauras $^{2}$

${ }^{1}$ Institut Jules Bordet, Haematology, Brussels, Belgium, ${ }^{2}$ Université Libre de Bruxelles, Ecole de Santé Publique, Brussels, Belgium, ${ }^{3}$ Institut Jules Bordet, Brussels, Belgium

Background: Patients with malignant hemopathies are increasingly treated by Oral Anticancer Medications (OAMs), including for bridging to transplant. The control of outpatients' adherence to treatment is a real challenge for clinicians. It has been demonstrated that poor adherence impacts the quality of response to treatment and the risk of relapse. Recent studies underline the link between adherence and patients' knowledge and beliefs, and also patientclinician interactions. Nonetheless, a key population group seems to be forgotten from research: Migrants and Ethnic Minorities (MEMs). MEMs have been identified as one of the most vulnerable groups in health care provision and recent findings underline considerable disparities and difficulties in communication between doctors and patients from different cultural and/or ethnic backgrounds. It seems therefore reasonable to anticipate a higher risk of poor adherence among MEMs.

Methods: Between 2012 and 2017, we conducted a 2 consecutive questionnaire-based survey which investigated 24 Healthcare Professionals' (HCPs) comfort and ability to take care for MEMs and 58 MEMs patients' satisfaction regarding their experience of transplantation. Subsequently, we conducted a scoping review of the scientific literature regarding MEMs' adherence to OAMs.

Results: First survey revealed that $76 \%$ of HCPs had difficulties to care for foreign patients and that the sole presence of Intercultural Mediation (IM) services was not enough to ensure a high quality of care for this population. Also, patients' satisfaction survey allows to highlight the strong presence of MEMs in our regular patients. Half of the participants were born abroad and $40 \%$ did not have French or Dutch as their mother language. Among those speaking little or not at all French, only a few were informed about 
the existence of IM services. MEMs' Satisfaction score was not lower. The results of the scoping review confirm unequal situations for MEMs in cancer care and highlights gaps in knowledge and research concerning the issue of MEMs' adherence. In order to reduce this gap, we created the MADESIO project, a two-phase approach. First phase will consist on developing some specific organizational supports, translating documents, facilitating the development of cultural competences in our staff and providing relevant tools to overcome urgent missing access to the Adherence Consultation and subsequent follow-up for MEMs. By using a mixed method approach, the second phase will evaluate MEMs' adherence and also explore the potential underlying mechanisms of poor-adherence for this population.

Conclusions: European migration has continued to grow rapidly in recent years. To eliminate the burden of health disparities and to ensure the best outcomes in MEMs with hematological malignancies, a collaborative approach between research fellows and clinicians is urgently needed. MADESIO's prospective approach of MEMs'care will contribute to overcome poor adherence. Also, we hope guarantee an equal access to innovative transplant procedures with an emphasis on inclusive care. Finally, the findings from research phase will be highly valuable for healthcare services to facilitate the development of patient tailored complex interventions and to guide providers in choosing best suited strategies to address and ultimately improve medication adherence.

Clinical Trial Registry: NA

Disclosure: Nothing to declare

\section{NO010 \\ Developing an inpatient physical activity intervention for patients undergoing haematopoietic stem cell transplantation}

\section{Lucy Ridgway}

\section{Oxford University Hospitals NHS Foundation Trust, Oxford, United Kingdom}

Background: The physical consequences of Haematopoietic Stem Cell Transplantation (HSCT) are well documented and include reductions in fitness, strength and energy, and delayed return to employment and sports. Evidence is growing that a targeted exercise intervention during the HSCT inpatient stay can ameliorate these losses, resulting in significant reductions in fatigue and improved quality of life on discharge and longer term (Van Haren 2013).
Reductions in the physical and psychological impacts of treatment may bring significant benefits in terms of quality of life, patient experience, and ability to return to work and other valued activities.

Methods: In order to explore the feasibility of providing an effective physical activity intervention within the confines of usual NHS care, we developed and piloted a programme, incorporating individualised exercise prescription and activity counselling. Screening tools to identify precautions or contraindications to exercise were developed to enable the intervention to be delivered by non-qualified therapy staff under the supervision of specialist physiotherapists, ensuring safety and cost-effectiveness.

The intervention was offered to thirty-five patients admitted for HCST between June 2017 and August 2018. Assessment included notes screening, blood counts, observations, activity history, physical outcome measures and activity preferences. Personalised plans were prescribed in collaboration with each patient and exercise diaries given. Advice was given regarding modifying exercise safely in response to change in blood counts during admission. All thirty five patients who were offered the intervention agreed to take part. Following the initial assessment, twenty five patients also received regular visits to adapt/progress the exercise program. Eight patients were subsequently reassessed at discharge with further activity counselling in preparation for home, repeated outcome measures, and patient feedback collected.

Results: There were no adverse events related to the intervention during the period of the project. Patient and staff feedback was excellent. The provision of a tailored physical activity intervention for patients admitted for HSCT appears safe, low-cost, and both acceptable and valuable to patients.

It is estimated that full delivery of this intervention (consisting of an initial assessment, three follow up visits a week, and a pre-discharge assessment) would require 7 hours of therapy assistant time over a four week admission, and cost approximately $£ 80$ per patient.

Conclusions: Exercise has been shown to be highly beneficial for patients undergoing HSCT. In order to prescribe exercise that is sufficiently challenging to elicit benefits, careful assessment is required to ensure both safety and efficacy. Incorporating personalised screening and exercise prescription within the project enabled exercises of significantly higher intensity to be offered, increasing efficacy and improving motivation. The model trialled within this project appeared to meet these needs effectively, with cost balanced pragmatically against the need for supervision by specialist physiotherapists.

We have demonstrated that a low-cost, evidence-based physical activity intervention can be delivered safely on an 
NHS inpatient haematology ward with minimal equipment and infrastructure required.

Disclosure: The author is not aware of any potential conflicts of interest.

\section{NO011}

Mental health is everyone's business: Addressing the psychological skills training needs of Haemato-Oncology research staff

\section{Surabhi Chaturvedi}

King's College Hospital, Haemato Oncology, London, United Kingdom

Background: Understanding the psychological state of patients with cancer is increasingly viewed as standard of care for all professionals with direct patient contact. Those coordinating clinical trials often have robust backgrounds in research sciences, but do not routinely receive psychological skills training. However, trial coordinators routinely interact with patients experiencing psychological distress. Patients entering clinical trials can be very vulnerable; undergoing randomisations to highly experimental therapies or receiving their final treatment option after treatment failures. A key role of mental health professionals embedded in multi- disciplinary teams is to offer training to colleagues to increase psychological skills to recognise their patients' emotional distress. Professional self-care, to recognise and look after own emotional needs resulting from regular contact with distressed patients, is another vital aspect of such training. A tailor-made psychological skills training session was piloted with members of a clinical trials team.

Methods: Two Haemato-Oncology psychotherapists delivered a three hour training session to 13 clinical trials staff. 4 were nurses, 9 were clinical-trial coordinators and research officers. The group had varied skills and understanding of psycho-social and interpersonal issues. To design the training around the groups' needs, facilitators invited participants to suggest topics of interest. The final schedule was an amalgam of participants' suggestions and facilitators' clinical experience on key concepts essential to an understanding of psychological issues. The session covered six key areas: 1. Adjustment and coping; 2. Common mental disorders such as anxiety and depression; 3 . Other forms of psychological distress; 4. Distinguishing normal adjustment from mental pathology; 5. Relational skills; 6. Vicarious trauma and self-care for professionals.

A 6-item (3 quantitative, 3 qualitative) evaluation form was used to gather feedback. To appraise this pilot session, qualitative comments generated rich data on participants' perceptions of the session.
Results: Quantitative results:

\begin{tabular}{llll}
\hline Item & \multicolumn{1}{c}{ Scale range } & Average score & Score range \\
\hline $\begin{array}{l}\text { a. Usefulness of the training } \\
\text { session }\end{array}$ & 9.10 & $8-10$ \\
$\begin{array}{l}\text { b. Likelihood of } \\
\text { recommending a training of } \\
\text { this type to peers }\end{array}$ & $1-10$ & 9.8 & $9-10$ \\
$\begin{array}{l}\text { c. Facilitator skills } \\
\text { (i) Clear explanations }\end{array}$ & - & & \\
$\begin{array}{l}\text { (ii) Maintaining } \\
\text { participants' interest }\end{array}$ & - & 4.5 & $3-5$ \\
$\begin{array}{l}\text { (iii) Responding to } \\
\text { questions } \\
\text { (iv) Deepening your }\end{array}$ & - & $4-5$ \\
understanding of \\
$\begin{array}{l}\text { psychological concepts } \\
\text { relevant to working with } \\
\text { patients }\end{array}$
\end{tabular}

[[NO011 Table] 1. Participant ratings of the training session]

Qualitative feedback: participants valued education on understanding patients' thoughts and feelings; understanding anxiety, depression and stress and their impact on thoughts and behaviours; coping strategies; emotional impact on staff and staff self-care strategies. Participants also appreciated the interactive and discussion-based format and the inclusion of practical, case-based examples. Participants provided feedback on areas they would like to focus on more in future training sessions, which included dealing with patients who may be in denial of their disease and further, in-depth training on professional self-care.

Conclusions: The session offers encouraging results in favour of delivering psychological skills training to professionals other than nurses and doctors who have a supportive, patient-facing role. A focus on balancing care of patients and on self-care as professionals is deemed extremely useful by participants.

Disclosure: Nothing to declare.

\section{NO012}

what about the psychological support in Italian bone marrow transplant centers participating in the GITMO network?

Gianpaolo Gargiulo $^{1}$, Iris Agreiter ${ }^{2}$, Cristina Mentone ${ }^{3}$, Stefano Botti ${ }^{4}$, Laura Orlando ${ }^{5}$, Barbara Cascone ${ }^{6}$

${ }^{1}$ University Hospital Federico II, Hematology, Napoli, Italy, ${ }^{2}$ St James's Hospital, HOHDC, Dublin, Ireland, ${ }^{3}$ Ospedale Mauriziano, Ematologia, Torino, Italy, ${ }^{4}$ IRCSS, Onco- 
Ematologia, Reggio Emilia, Italy, ${ }^{5}$ Istittuto Europeo di Oncologia, Onco-Ematologia, Milano, Italy, ${ }^{6}$ Healh Social Forum Association, Patient Care, Sorrento, Italy

Background: Patients undergoing hematopoietic stem cell transplantation (HSCT) are frequently exposed to psychological distress due to long hospitalization, isolation and complex care. The coping process is determined by the severity of the diagnosis, the therapeutic protocol, by patient's familiar and supportive network and by personal resources in challenging situations as HSCT. An adequate psychological support is essential for all different phases of transplant, in order to promote the success of treatment and achieving a good quality of life. The 7th edition of JACIE recommends to have a psychologist within each HSCT team.

Methods: The Italian HSCT centers belonging to the GITMO network were invited to participate to an online survey in order to verify the current availability and the organizational modalities of the psychological support offered to patients, caregivers and health personnel, which are particularly exposed to a burn-out risk. A specific questionnaire elaborated and validated by the GITMO nurses board was administered through the Google Drive platform. The self-report questionnaire was divided into 26 items. The main topics included the psychological support modality offered to patients, psychological support to caregivers and health care personnel, psychological education of healthcare staff on specific aspects of HSCT.

Results: Eighty centers were invited to participate, 64 replied $(80 \%)$ which are divided into 12 pediatric, 5 mixed and 47 adult centers. Eleven centers declared to perform autologous transplants, while 53 centers perform autologous, allogeneic, matched unrelated donor (MUD) and haploidentical transplants. The psychological support provided to patients is available in $94 \%$ of the centers, in particular in $91 \%$ of the pediatric centers, in all the mixed centers (100\%) and in $93 \%$ of the adult centers.In 29 centers the psychologist is part of the staff of Hematology and HSCT Unit, in 16 cases part of the Oncology/Hematology Department, while in 15 cases the psychologist belongs to the Hospital's Psychotherapy Department.In 17 centers interviews with the psychologist are performed by default, while in 24 centers they are activated exclusively for patients with obvious and serious mental disorders; in 7 centers the psychologist is present every day of the week. The most widely used psychological support activities are interviews, narrative medicine, art therapy, shiatzu massages, music therapy, games, karate, hypnosis, clowns, visualizations, autogenic training, reiki, yoga, makeup projects for women.

Conclusions: The data show that in most centers the psychological/psychotherapeutic support appears not to be sufficiently structured in order to respond adequately to the needs of the patients. The required support, which is needed during the different phases of HSCT is not always achievable. Psychologists, psychiatrists and supporting procedures need to be defined with a greater importance, JACIE is a good start point to implement them as part of the standard HSCT team.

Disclosure: Nothing of declare

\section{NO013}

Discharge following hematopoietic stem cell transplant (HSCT): What problems are patients taking home?

\section{Sarah Liptrott ${ }^{1}$, Margherita Clerici, ${ }^{1}$ Laura Orlando $^{1}$, Dimitrios Protogiros ${ }^{2}$, Tiziana Suardi, Corrado Tarella, Giorgio Magon ${ }^{1}$}

${ }^{1}$ IEO, European Institute of Oncology IRCCS, Milano, Italy, ${ }^{2}$ Department of Nursing, School of Health Sciences, National and Kapodistrian University of Athens, Athens, Greece

Background: Patients undergoing SCT are often discharged with ongoing side effects from treatment felt to be manageable at home. To facilitate this, nurses must provide appropriate education for patients and caregivers to empower them to manage issues after discharge. Our aim was to identify the nature and extent of problems faced by patients at discharge.

Methods: We performed a retrospective analysis of nursing records to identify the presence and grade of Nursing Sensitive Outcomes (NSO's) at discharge using the Oncology Nursing Minimum Data Set (Milani et al., 2013). Patients having undergone autologous or allogeneic SCT between 01/07/2017 and 30/06/2018 and discharged from the SCT unit at single centre in Italy, were eligible for inclusion. Data collection included demographic and clinical characteristics.

Results: The characteristics of 41 patients are presented in Table 1 . The majority of patients were male $(n=28$, $68.3 \%$ ) and had undergone autologous SCT (autoSCT). The mean number of active NSO's per patient was 4.8 (SD \pm 1.68 - range 0-7), with minimal differences between autoSCT and allogeneic SCT $(5.11+1.67,4.21+1.58)$. Only 3 patients had no ongoing NSO's identified at discharge. The most commonly reported NSO's included bone marrow suppression: anemia $\mathrm{n}=33$ (autoSCT $\mathrm{n}=23$ $(85.2 \%)$, alloSCT $\mathrm{n}=10(71.4 \%)$ ), thrombocytopenia $\mathrm{n}=$ 21 (autoSCT $\mathrm{n}=23(85.2 \%)$, alloSCT $\mathrm{n}=8(57.1 \%)$ ), and leucopenia $\mathrm{n}=18$ (autoSCT $\mathrm{n}=8(29.6 \%$ ), alloSCT $\mathrm{n}=6$ $(42.9 \%))$. Grade 3 or $4(\mathrm{G} 3 / 4)$ events had similar distributions between SCT types (autoSCT 51.9\%, alloSCT 
42.9\%). 27 patients had mucositis (autoSCT $\mathrm{n}=20$ $(74.1 \%)$, alloSCT $n=7(50.0 \%))$, the majority G1. Fatigue was seen in 26 patients (autoSCT $\mathrm{n}=17(63.0 \%)$, alloSCT $\mathrm{n}=9(64.3 \%))$, the majority being G1 $(\mathrm{n}=22)$. G1-2 appetite loss was identified in 8 patients (autoSCT $\mathrm{n}=5$ $(18.5 \%)$, alloSCT $\mathrm{n}=3(21.4 \%))$. Other NSO's reported $(\mathrm{n} \leq 3)$ included localised edema (G3), glucose intolerance (G⿳亠丷厂 - diabetic patient), nausea, cough, constipation, cutaneous rash, hypertension, skin ulcer, and dehydration Grades 1 - 2. All patients were autonomous in Instrumental Activities of Daily Living (IADL), whilst one patient required assistance with ADL's - this patient had a wound infection following alloSCT and was discharged with a vacuum drain insitu. 5 alloSCT pts $(30.8 \%)$ were evaluated as autonomous in relation to treatment understanding versus $63.0 \%$ in the autoSCT group. Complete autonomy in relation to management of medical devices (e.g. central venous catheters), nutrition and pain were only achieved in 50-60\% of autoSCT patients and 40-50\% of alloSCT patients. Risk of falls was reported at discharge for 4 patients (all alloSCT). Two patients scored a significant value of $7 / 10$ on the Distress Scale at discharge (autoSCT and alloSCT).

Conclusions: This data has shown a significant number of patients have a range of NSO's still ongoing at discharge and many patients were not completely autonomous in aspects of self-care. These findings could be used to review nurse's current education practices with patients and caregivers in preparation for discharge. Follow up of ongoing NSO's and addressing the issue of incomplete autonomy in areas of self-care should also be investigated and may warrant continued support.

Disclosure: Nothing to declare

\section{NO014}

Abstract already published.

\section{NO015}

Abstract already published.

\section{NO016}

How does allogeneic stem cell transplantation affect patients sex life and how are sexual issues addressed in clinical care?

\author{
Solveig Adalsteinsdottir ${ }^{1}$, Carolina Competisa ${ }^{2}$ \\ ${ }^{1}$ Karolinksa University Hospital, Celltherapy and \\ Allogengic Stamcell Transplantation, Huddinge/Stockholm, \\ Sweden, ${ }^{2}$ Ryhov Hospital, Jönköping, Sweden
}

Background: Cancer patients that undergo allogeneic stem cell transplantation risk side effects that may affect their sex life. Earlier studies show that cancer patients who undergo stem cell transplantation are not satisfied with the information they receive about possible treatment-induced sexual health problems that can occur. The purpose of this study was to identify to what extent patients, that had undergone allogeneic stem cell transplantation, received information about the possible negative impact on their sex life, what sexual problems they acquired and up to what degree they have experienced them.

Methods: A quantitative cross-sectional study. A questionnaire, based in half on the PROMIS SexFS v2.0, was sent out to 92 participants. This included all persons over 18 years of age that had underwent an allogeneic stem cell transplantation due to a malign hematological disorder between the years 2012 and 2014 at a university hospital in Sweden and could read and write Swedish.

Results: Sixty-three former patients consented participation (response rate was $68 \%$ ). The average age was 57 (range between 20 and 75). Most of the participants (87\%) had received little or no information about how their sex life could be affected by the treatment. Almost half of the participants wanted this information (43\%). Many of those who answered the questionnaire had been sexually active in the last 30 days before answering (81\% men vs. $63 \%$ women) and about half of them enjoyed their sex lives (40\% men vs. $63 \%$ women). The most common problem reported by men was erectile dysfunction (42\%) and for women, vagina dryness $(69 \%)$.

Conclusions: Information about the possible effects of an allogeneic stem cell transplantation on sexual health is lacking. About $40 \%$ of the men and $70 \%$ of the women reported that they had at least one sexual problem two to four years after the treatment. Based on this study we have identified the importance of discussing the subject in all stages of the process. We believe, based on the results of this study, that open conversations, free of prejudice, about the possible side effects on the patients sex life is very important for the patient.

Disclosure: Nothing to declare

\section{NO017}

\section{Managing electrolytes during apheresis}

\section{Daniela Kulik ${ }^{1}$, Natalie Hollis ${ }^{2}$}

${ }^{1}$ University Hospitals Birmingham, Apheresis/ Haematology, Birmingham, United Kingdom, ${ }^{2}$ University Hospitals Birmingham, Apheresis, Birmingham, United Kingdom 
Background: Stem Cell collection procedures are reasonably safe for the majority of patients. However, it is recognised that there are some side effects with the procedure, including citrate toxicities (Babic and Trigoso, 2017). During stem cell collection, patient's calcium levels can decrease due to the anticoagulant (in this case Anticoagulant Citrate Dextrose Solution- Solution A) used to prevent blood clotting in the apheresis system. Previous audits at a local level have shown that this decrease can be corrected by pre-emptive oral calcium administration (Wong and Bird, 2017). The purpose of this audit was to collect and analyse data to ascertain if any other electrolytes were affected during the procedure and to see if patients would benefit from similar interventions. Furthermore, anecdotal evidence suggested that despite pre-emptive oral calcium administration, some patients remain symptomatic therefore it was deemed important to investigate other causes.

Methods: We collected data from 154 patients between January and December 2017 who attended the apheresis unit for stem cell collection. Exclusion criteria consisted of patients results that were not available due to lab or procedural errors. As per our Standard Operating Procedure (SOP), we took blood samples for urea and electrolytes from patients immediately before and after each stem cell collection (Wong, 2017). They were sent to our internal biochemistry laboratory for analysing and the results were viewed on our prescribing information and communication system (PICS). The blood results were then recorded on an excel spreadsheet for analysis and compared with available data and evidence to underpin future practice.

Results: We analysed data from day one of stem cell collection for calcium, potassium and magnesium levels, prior to any infusion of saline to avoid dilution of samples. Despite pre medication and control of symptoms with oral calcium, $17 \%$ of patients still dropped calcium levels. In addition, although $80 \%$ of patients had a fall in magnesium levels, this decrease in magnesium was not significant. The most important finding was that $95 \%$ of patients had a decrease in potassium levels after just one day of stem cell collection, with an average drop of $15.4 \%$. Furthermore, $67 \%$ of all patients had a potassium level below normal local laboratory levels post 1 day of stem cell collection, which would suggest oral replacement may be indicated.

Conclusions: This audit has shown that calcium levels are effectively corrected during stem cell collection through administration of oral calcium pre procedure (Wong and Bird, 2017). Also, the majority of the patients had a drop in potassium and magnesium levels, which has lead our unit to look at changes in our practice. We are now discussing with the medical team the need for oral replacements to be given to patients to take home after the procedure.

Clinical Trial Registry: N/a

Disclosure: Nothing to declare

\section{NO018}

The nursing assessment of patients undergoing allogeneic stem cell transplantation through the narrative based medicine a initial study of a single centre

\section{Giuseppa Cusumano, Lucrezia Imbalzano, Tayan Trimarco, Antonio Maria Rossetti, Fabio Provenzano, Massimo Martino}

Grande Ospedale Metropolitano BMM, CTMO, Reggio Calabria, Italy

Background: The Nursing assessment of patients undergoing allogeneic stem cell transplantation (AlloSCT), is often limited to the clinical symptoms, without an overall view of the patient's path, usually very long and complex. Narrative based medicine (NBM) gives the possibility to identify the criticalities and the strength perceived by the patient to improve compliance in the future and to facilitate the healing and recovery.

Methods: We used a semi-structured interview applying an uniform method of evaluations of the patient tale, and focusing the attention on aims faced by the patient and by the family.

The main points are: Discovery of the disease (how, when); Therapeutic program (chemotherapy, duration, side effects, family care); Waiting Period; Transplantation Phase; and Life After Transplantation (psychological, social and family implications).

Patients have described their experience of care. The tale recorded has been divided into periods with its encoding. It was elaborated a coded database for each item, to allow a homogeneous evaluation of the data, by splitting the items to social, family and welfare implications and also was made the counting of words and the most repeated ones.

Results: We interviewed 15 patients undergoing an AlloSCT. The interviews had an average duration of 15,75 minutes and 673 words were used and the most widely used were doctors, transplantation, nurses, disease, alone. $80 \%$ report a good communicative relationship with the physicians and only $60 \%$ with the nurses. But in contradiction $100 \%$ of the patients report that the support given by both categories was adapted to the treatment of his illness. $60 \%$ of patients need the caregiver to help him in taking drug therapy after discharge. While in the psychological context, $67 \%$ declared that their life has changed for better, giving them a positive perspective of the daily moments. Patients who performed the transplantation a long time ago had less difficulty in telling their experience, and were more rational and detailed in talking about the difficult times. While the stories of the patients who underwent AlloSCT more recently, were more laden with emotional 
moments. It was possible to evaluate the relationships: patient/nurses, patient/physician, patient/family and the importance of collaboration between these figures and communication with the patients noted.

Conclusions: Preliminary results of a study indicate that NBM can be an important tool to assess the effectiveness of the patient's overall care and suggest that an integration of narrative and scientific approaches could be useful in order to improve therapies' effectiveness.

Disclosure: nothing to declare

\section{NO019}

Peripheral insertion central catheter (PICC) in the management of acute leukemia and stem cell transplant patients - feasibility and outcomes

Marcia Valentim $^{1}$, Luzinete Rangel ${ }^{1}$, Andreia Assis ${ }^{I}$, Alice Pissiali $^{1}$, Marcia Garnica ${ }^{2}$

${ }^{1}$ Complexo Hospitalar de Niteroi CHN, Transplante, Niterói, Brazil, ${ }^{2}$ Universidade Federal do Rio de Janeiro, Clinica Medica - Faculdade de Medicina- Professora Adjunta, Rio de Janeiro, Brazil

Background: Patients submitted to acute leukemia treatment and stem cell transplant recipients need a safe and prolonged vascular access since they experience prolong and consecutives hospitalizations. Long term venous catheters confer comfort for these patients. These catheters have been associated to few episodes of infection compared to short-duration venous catheters. Peripheral insertion central catheter is a long-term catheter with a peripheral insertion, not expensive, that can be easily inserted by a trained nurse. In this study, we describe our experience with PICC in leukemia patients and in stem cell transplant, in terms of feasibility and outcomes.

Methods: Prospective cohort performed in single center including acute leukemia patients (lymphoid or myeloid) in induction remission or intensification cycles of chemotherapy and patients submitted to autologous or allogeneic stem cell transplant. PICC implantation has been performed by a trained nurse group by ultrasound guided insertion. For Leukemia patients, the suggested routine has been PICC as first choice long-term catheter, implanted in the first hospitalization for chemotherapy and remained inserted until recovery of the last chemotherapy cycle, including outpatient periods between cycles. For transplant patients, the recommendation has been PICC insertion for autologous (before condition regimen) until discharge after hematopoietic recovery. For allogeneic patients, the central venous first choice is semi-implantable long-term catheter, inserted before conditioning. If there is necessity of catheter removal, a PICC insertion has been recommended. There is no recommendation based on duration of PICC insertion for removal. Removal indications have been: thrombosis, suspected or documented infection related to PICC, or discontinuation of a vascular access necessity. For these analyses, data from patients treated from 2015 to 2017 were selected.

Results: A total of 57 hematological patients (including 35 leukemia patients) and 47 (36 autologous and 11 allogeneic) transplant recipients had at least one PICC in placed during the study. Regarding leukemia patients, the median duration of PICC was 23 (ranging from 1 to 365) days; in seventeen (49\%), PICC was maintained for more than 50 days; and only $14(40 \%)$ needed PICC replacement during treatment. Regarding autologous recipients, the median duration of PICC was 14 ( 1 - 32) days; only 4 $(11 \%)$ needed PICC replacement, and removal criteria was mainly hematopoietic recovery and discharge. One patient $(3,3 \%)$ developed blood stream infection related to venous catheter and removal was indicated. Eleven allogeneic transplant patients had a PICC installed during the study, all indicated as a replacement long-term catheter. The median PICC duration was $42(1-442)$ days.

Conclusions: In our cohort, indication of PICC as a venous catheter for leukemia and transplant patients was feasible and safety. There were few replacements and also few episodes of infection related to the vascular device. PICC was able to support intense chemotherapy infusions, high density of blood cells necessity and also to management of other complications related to chemotherapy. The insertion was easily performed by trained nurses, in hospitalization-rooms.

PICC is an interesting choice as venous catheter in leukemia and stem cell transplants.

Clinical Trial Registry: Not applicable

Disclosure: "Nothing to declare"

\section{NO020 \\ Evaluation of sleep quality in hematopoietic stem cell transplantation patients}

\section{Seckin Erdal, Aysem Kuni, Sevinc Ince, Kiymet Hakli, Azize Guneri}

Acibadem Altunizade Hospital, Adult Bone Marrow Transplantation Unit, Istanbul, Turkey

Background: Hematopoietic Stem Cell Transplantation (HSCT) might have early and late complications as well as social, economic, physiological and psychological problems. One of the major problem is sleep disturbances. Because of the sleep disturbances symptoms such as; 
fatigue, discomfort, decreased attention, increased sensitivity to pain, confusion, irritability, irrational thoughts, hallucinations and lack of appetite may occur. For this reason, sleep is seen as an important variable of health, affecting the quality of life and well-being of the individual. Maintaining and improving the quality of life of the patients is the responsibility of the nurses and it is important that nurses can develop a care plan for sleep problems that affect quality of life. The aim of this study is to evaluate sleep quality of the patients who are undergoing hematopoietic stem cell transplantation, prevent the problems that may be faced according to outcome of the research and determine the nursing approaches in order to prevent for problems.

Methods: This prospective study was performed between January 2018 and June 2018 in the adult bone marrow transplantation unit of a private hospital after obtaining the approval of the ethics committee. The study sample consisted of 56 patients who underwent stem cell transplantation. The Pittsburg Sleep Quality Index (PSQI) form was completed by the researchers when hospitalized and discharged for the same patients. For statistical analysis, IBM SPSS Statistics 22 program used descriptive statistical methods (mean, standard deviation, frequency) as well as Shapiro Wilks test, Mann Whitney U test, Kruskal Wallis test, Friedman test, Wilcoxon Signed Ranks test.

Results: A total of 59 patients $44.1 \%(\mathrm{n}=26)$ were female, $55.9 \%(\mathrm{n}=33)$ were male. The majority of the patients were $42,4 \%(n=25)$ between $45-65$ years old. The scale has been used four times during the hospitalization. According to the distribution of PSQI total scores on the administration day $42.4 \%$, first week $50.8 \%$, second week $\% 50,8$ and on the third week $\% 61,5$ of the patients had poor sleep quality. After the discharge, PSQI total scores indicated that at the first week $\% 32,2$, second week $\% 27,1$ and the third week $\% 25,4$ of the patients had poor sleep quality.

During the hospitalization period poor sleep quality significantly increased. After the discharge, sleep quality was poor in the first week but it was started to improve by staying at home. Addionally, during the period of hospitalization, it was determined by the effect of the environmental factor, such as ventilation sound, pump sound, treatments and nurses who enters to the room were detrimental on patient's sleep quality.

Conclusions: It was observed that sleep quality was significantly impaired during the period of hematopoietic stem cell transplantation. For this reason, it is important to get information on the history of sleep quality and clinical follow-up by nurses when transplant patients are admitted to the clinic. Improving the care plan for the nurses on the sleep problems can positively affect the quality of life of the patients.

Disclosure: Nothing to declare

\section{NO021}

Nursing care experience of caregivers of patients after hematopoietic stem cell transplantion: A qualitative study

\section{Si Cheng, Yun Fang}

Union Hospital affiliated with Tongji Medical College, Huazhong University of Science and Technology, Department of Hematology, Wuhan, China

Background: To explore the nuring care experience of caregivers of patients after hematopoietic stem cell transplantation.

Methods: There were11 caregivers of patients after hematopoietic stem cell transplantation evaluated by using semi-structured interview, phenomenological study method, and data analyzed according to Colaizzi phenomenon analysis method.

Results: Four main themes were extracted including complex psychological feelings, lack of knowledge and skills, negative effects of disease, eager for social, family and medical support.

Conclusions: Clinical professionals need to pay attention to the physical and mental experience of the caregivers, while nursing patients after hematopoietic stem cell transplantation, to get to know physical and mental reactions of caregivers, as well as care requirements through knowing their nursing care experience. So clinical professionals can establish a personalized care program at the time when patients are hospitalized, helping promote the physical and mental health of caregivers and improve their nursing care ability,thus to establish good psychological adjustment, and to promote recovery and improve their quality of life.

Disclosure: Nothing to declare.

\section{NO022}

perception of patients about the insertion of central venous catheter: preparation, functionality and body image - study preliminary results

\section{Cristina Canaleta Ros, Noelia Arranz Padilla, Susana Cortes Barba, Merce Triquell Garrell, Anna Camon Yarto, JManel Portos, Silvana Novelli, Irene Garcia Cadenas, Iria Gonzalez Alvarez, Anna Barata, Karla Salas, Jorge Sierra}

Hospital Santa Creu i Sant Pau, Barcelona, Spain

Background: Central venous catheter is necessary in a large part of onco-hematological treatment. Its placement is 
not free of risks and carries concern for patients. However, few studies have evaluated the patient perception about this practice.

Therefore, the aim of this study is to investigate how patients face the insertion of a $\mathrm{CVC}$ and the impact it has on their functionality and body image.

Methods: A descriptive, longitudinal study that consecutively included subjects with hematologic neoplasm who were up to 7 days after the insertion of a CVC to receive intensive chemotherapy. In the absence of specific tools for the purpose of the study, a questionnaire of 26 items grouped into the following constructors was created: 1) Insertion of the CVC: evaluates information received, pain, emotional state; 2) Comfort of the CVC: examines the interference of the CVC in daily activities; 3) Body Image and 4) Functionality at home. Each item was scored from 0 to 3 points.

In the construction of the questionnaire, 10 patients were invited to complete and / or reject the items raised and confirm their correct understanding. Clinical variables of the medical history were obtained. Descriptive analyses, the Student's T test, and the study of nonparametric correlations were performed to determine potential differences between the study parameters according to sex and age.

Results: 32 patients were included; $18(56.3 \%)$ men, median age of 53 years (range 17-70), 26 (81.3\%) carriers of central venous catheter and $6(18.8 \%)$ of central venous catheter of peripheral insertion (PICC). For $83 \%$ of the patients $(\mathrm{n}=25)$ the information received about the insertion of the CVC was sufficient and satisfactory. $29 \%(\mathrm{n}=9)$ reported significant pain associated with the procedure of insertion. Analgesic treatment was effective in $84 \%$ of the cases $(\mathrm{n}=26)$. The $\mathrm{CVC}$ significantly interfered in personal hygiene $(n=30,94 \%)$ and in sleep $(n=26,81 \%)$. The visibility of the CVC was negatively evaluated in 16 patients (50\%), and $14(43.7 \%)$ reported hiding the CVC. $16 \%(\mathrm{n}=5)$ expressed regretting inserting a CVC. Men and women did not differ in the experience of insertion $(p=0.216)$, comfort $(p=0.406)$ and impact on body image $(p=0.799)$. Older patients described better experience in terms of insertion $(\mathrm{r}=0.337)$, obtaining a tendency towards significance $(p=0.059)$. The youngest patients showed lower comfort $(r=-0.244, p=0.178)$ and greater affectation of the corporal image $(r=-0.306)$, tending the preceding to a statistical significance $(\mathrm{p}=0.088)$.

Conclusions: Preliminary results suggest that the insertion of a CVC involves significant discomfort, highlighting the visibility and interference on daily activities. The ongoing expanded recruitment and its future analysis will allow understanding of potential differences in the experience of insertion, functionality and body image and will delimit groups to adjust interventions and optimize the experience of the CVC.

Disclosure: Nothing to declare 